UNIVERSUM $\cdot$ Vol. $33 \cdot \mathrm{N}^{\mathrm{o}} 2 \cdot 2018 \cdot$ Universidad de Talca

La Física contra Tomistas de Lorenzo Romo S. J.: apuntes sobre una controversia filosófica en el Chile

del siglo XVIII

Abel Aravena Zamora

Pp. 53 a 73

\title{
LA FÍSICA CONTRA TOMISTAS DE LORENZO ROMO S. J.: APUNTES SOBRE UNA CONTROVERSIA FILOSÓFICA EN EL CHILE DEL SIGLO XVIII ${ }^{1}$
}

\author{
Lorenzo Romo's Physica contra Thomistas: \\ notes on a philosophical controversy in XVIIIth century Chile
}

\author{
Abel Aravena Zamora*
}

\begin{abstract}
RESUMEN
Este artículo expone los aspectos generales más relevantes de la controversia filosófica presente en el curso manuscrito Física contra Tomistas (1738) del maestro jesuita Lorenzo Romo (1703-1768). Esta obra inédita, dictada en el Colegio Máximo San Miguel y conservada por la tradición filosófica con un título errado, es testimonio de la disputa teórica que el jesuita sostiene con los autores tomistas en torno a determinados temas filosóficos que devienen de las doctrinas aristotélicas. Presentamos primero una breve nota biográfica del autor y algunas informaciones sobre sus obras conservadas en la actualidad. Luego, analizamos especialmente el suarecianismo expresado en la
\end{abstract}

\footnotetext{
${ }^{1}$ El autor agradece enormemente a Soledad Díaz de los Reyes, del Archivo Histórico Mercedario de Santiago de Chile (AHME), por su valiosa colaboración en el desarrollo de este trabajo.

* Investigador Asociado Centro de Estudios Históricos, Universidad Bernardo O’Higgins. Santiago, Chile. Miembro grupo de investigación Discursos legitimadores de la conquista y colonización de América al norte y al sur del continente, Universidad de Alcalá, España. Correo electrónico: abel. aravena.zamora@ gmail.com
}

Artículo recibido el 08 de marzo de 2018. Aceptado el 24 de julio de 2018. 
concepción metafísica de la materia prima y en la controversia sobre la distinción entre esencia y existencia. Para ello, ofrecemos la traducción al castellano de los pasajes que hemos juzgado más relevantes. De este modo, pretendemos rescatar y visibilizar el patrimonio filosófico del país $\mathrm{y}$, en consecuencia, posibilitar nuevas investigaciones en el área y, en particular, en torno a la figura y el quehacer filosófico de este maestro jesuita.

Palabras clave: escolástica americana, controversias filosóficas, filosofía colonial chilena, suarecianismo, Lorenzo Romo.

\begin{abstract}
This paper aims to expose the most relevant general aspects of the philosophical controversy presented in the manuscript course Physics against Thomists (1738) by the Jesuit Master Lorenzo Romo (1703-1768). This unpublished work, given at the Colegio Máximo San Miguel (Santiago de Chile) and preserved by the philosophical tradition with a wrong title, is testimony of the theoretical dispute that the Jesuit maintains with Thomist authors about certain philosophical issues that come from the Aristotelian doctrines. First we present a brief biographical note of the author and some information about his works currently conserved. Then, we analyze especially the Suarecianism expressed in the metaphysical conception of the materia prima and in the controversy about the distinction between essence and existence. To that end, we offer the translation into Spanish of the passages that we have deemed most relevant. In this way, we intend to rescue and make visible the philosophical heritage of the country and, consequently, enable new research in the area and, in particular, around the figure and philosophical work of this Jesuit teacher.
\end{abstract}

Keywords: American Scholasticism, Philosophical Controversies, Chilean Colonial Philosophy, Suarecianism, Lorenzo Romo.

\title{
Introducción
}

La actividad filosófica durante el Chile de la época colonial es aún un área muy poco explorada. En general, se desconocen casi totalmente las fuentes primarias conservadas en diferentes archivos, siendo muy pocos los autores y las obras conocidas del período. Más precisamente, el vacío es enorme con respecto al desarrollo y tratamiento de los contenidos de los cursus, pues, salvo contadas excepciones, son escasos los estudios que aborden esta materia. En este sentido, el conocimiento de la tradición filosófica chilena está aún incompleto. 
Los esfuerzos pioneros en esta área se centraron en identificar y catalogar las obras conservadas, sus autores y las tendencias filosóficas cultivadas ${ }^{2}$. Con respecto a esta obra en particular de Romo, hay que señalar que ha sido descrita brevemente por los jesuitas Walter Hanisch (1963, p. 66) y Fernando Astorquiza (1982, p. 42), quienes, sin embargo, la identifican erróneamente con el título

Física Tomista, mientras que Walter Redmond solo la identifica con el título de Física (1972, p. 84).

Por esta razón, en este artículo pretendemos presentar aquellos aspectos generales más relevantes de la obra y profundizar, especialmente, en algunas aristas de la disputa teórica que el jesuita sostiene con los autores tomistas en torno a determinados temas filosóficos. Para ello, ofrecemos los textos que hemos juzgado relevantes y, para hacer más comprensible este trabajo, entregamos nuestra traducción al castellano de algunos otros pasajes.

Así, pretendemos, por un lado, contribuir en la generación de nuevas investigaciones en esta área, y, particularmente, en torno al quehacer filosófico del jesuita Lorenzo Romo; y, por otro, rescatar y visibilizar el patrimonio filosófico nacional.

\section{Nota biográfica}

El nombre de Lorenzo Romo (Andalucía, 1703-1768) resulta casi desconocido dentro de la historia de la filosofía chilena. Las breves noticias biográficas que hallamos en los catálogos sobre los personajes destacados de la Compañía en la época colonial informan escasamente sobre su actividad filosófica en estas latitudes.

Según aquellos registros, ingresó a la Compañía de Jesús el 3 de marzo de 1720 y emitió sus últimos votos en 1737. Luego, viajó a Chile con una de las expediciones de misioneros, siendo profesor en el seminario de Concepción, rector y maestro de novicios en Santiago y más tarde, en 1751, rector del Convictorio San Francisco Javier. El Padre Enrich S. J. rescata información de alto interés sobre su perfil docente:

Fue hombre de baja estatura, pero de altos pensamientos; de pocas palabras, pero de mucha sabiduría: la cual no sabía ostentar en las conversaciones, pero sí en la cátedra; que fue su principal ministerio. Puesto en ella, con los ojos cerrados, como si durmiera, dictaba las lecciones de teología a sus discípulos, sin más papel que algunas citas de los autores más afamados.

\footnotetext{
${ }^{2}$ Cf. Aravena Zamora, 2015; Hanisch, 1963; Quiles, 1953.
} 
Sólo hablaba con los seglares cuando lo requería la dirección espiritual de sus penitentes; en los cuales resplandecía comúnmente su espíritu"3. Similar testimonio puede hallarse también en la pluma de Hanisch (1963, p. 63).

Hacia la época de la expulsión de la Compañía de Jesús, Romo se encontraba enfermo en Santiago, no obstante, rehusó quedarse en Chile, rechazando la indulgencia del Gobernador del Reino, y decidió seguir a sus hermanos y morir en su compañía. Así, -según relata Enrich- falleció en 1768, durante el viaje al destierro, sin recibir los santos sacramentos ni tener quien le auxiliase en la última hora. Por ello, su cuerpo fue arrojado al mar, que, a pesar de haber estado muy embravecido, se calmó al momento 4 .

\section{Los cursos filosóficos}

Entre las obras filosóficas coloniales, conservadas en su gran mayoría en el Fondo Antiguo del Archivo Nacional Histórico y, en menor medida, en los archivos de las diversas órdenes religiosas, destaca la labor de Lorenzo Romo, pues contamos con dos de sus comentarios filosóficos. Llama la atención, sin embargo, que ambas obras se resguardan en archivos diferentes de la ciudad: los comentarios a la Lógica, se encuentran en el Archivo Jesuita, mientras que la Física contra Tomistas se conserva en el Archivo Histórico Mercedario.

De acuerdo con sus fechas, ambas obras fueron dictadas en el Colegio Máximo de San Miguel, donde Romo enseñaba filosofía desde 1738, tras poco tiempo de llegar a Chile. Vale la pena recordar que dicha institución había pasado a ser Universidad Pontificia en 1621, obteniendo la facultad para entregar títulos en Filosofía y Teología, los cuales eran válidos únicamente en las Indias ${ }^{5}$.

Desde un punto de vista material, ambos volúmenes comparten muchas características. Pues, tanto los aspectos relativos a los materiales de confección de los códices (encuadernación en pergamino rústico, elaboración de cuadernillos, inscripción en el lomo de los volúmenes, calidad del papel y de la tinta) como los referidos a sus aspectos formales constitutivos (estructura y organización de contenidos, aspecto general, ángulo, diseño y trazado de la letra) coinciden indudablemente.

\footnotetext{
${ }^{3}$ Enrich II (1891, p. 413). Cf. también Enrich 2/J/294 carp. 17, pp. 11-13.

${ }^{4}$ Cf. Tampe (2008, p. 225); Astorquiza (1982, p. 42); Hanisch (1963, p. 63) y Enrich II (1891, p. 413 ss).

${ }^{5}$ Para mayor información sobre el Colegio Máximo San Miguel, véase Contreras (2014, pp. 40-44); Guarda (1978, pp. 218-219); Hanisch (1974, p. 42; 1963, pp. 33-34) y Jobet (1970, p. 26).
} 
Como hemos avanzado, el curso de Lógica (1738), se resguarda en el Archivo Jesuita. Esta obra da cuenta de los contenidos estudiados durante el primer año de los estudios de filosofía o cátedra de Artes $^{6}$. El manuscrito posee 189 folios, está escrito a línea tirada, íntegramente en latín y sus contenidos se dividen en cinco libros: el primero aborda las Sumulas; el segundo, los elementos introductorios a la disciplina (prooemialibus logicae); el tercero estudia los universales (De universalibus et rerum distinctione); el cuarto se centra en las especies de universales (De universali in specie); y el quinto, en el concreto y abstracto, el signo y la demostración (De concretis et abstractis, de signo et demonstratione). Grosso modo, en el primer libro estudia la división y propiedades del término lógico; la definición, división y reglas entre los distintos tipos de modos de saber (modus sciendi); los distintos aspectos de la proposición y, por último, se centra en el tema de la argumentación. En el segundo, aborda la naturaleza y las propiedades de la lógica y su concurso y necesidad para adquirir las otras ciencias; en el tercero y cuarto estudia el tema de los universales; mientras que en el último analiza cada uno de los elementos enunciados.

Por otra parte, la Fisica contra Tomistas (1739) se halla actualmente, con identificación Ms. 1335, en el Archivo Histórico Mercedario. El volumen presenta, en general, un buen estado de conservación. Tiene unas dimensiones de $22 \times 16 \times 3 \mathrm{cms}$, contiene 228 fojas de texto sin numerar y no conserva su portada inicial. Está encuadernado en pergamino rústico, su portada está rasgada del lomo, con bastante suciedad y deformada por la humedad y los cambios de temperatura. Está compuesto por 27 cuadernillos de papel, escrito con tinta ferruginosa a línea tirada y tiene entre 44 y 46 líneas de texto por hoja. El estado de conservación de las fojas es bastante bueno, sus contenidos son legibles, y no han perdido su soporte, por lo que el volumen puede manipularse sin riesgo de dañarlo.

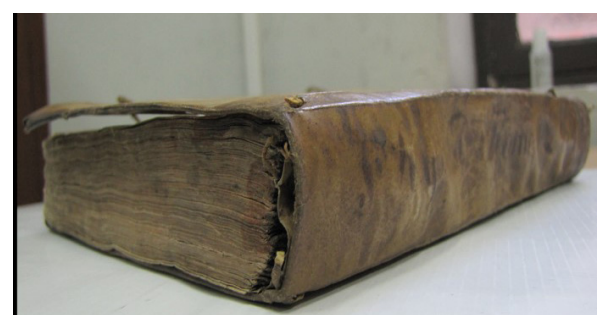

Detalle de la encuadernación de la imagen

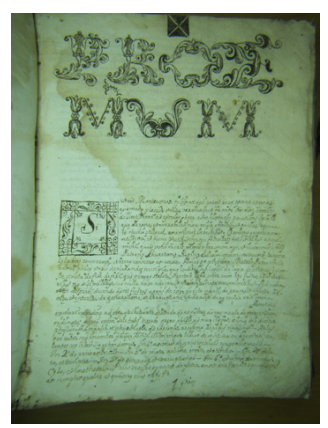

Detalle, f. 1 r.

${ }^{6}$ Cf. Skariça (2010, pp. 9-10). Respecto a otros cursos de la época en Chile, véase Aravena Zamora, 2016a y 2016 b. 
Los contenidos se estructuran en seis libros, que se subdividen a su vez en un número variable de disputaciones y secciones, además de un proemio inicial. Vale la pena mencionar que esta forma de abordar las materias, en seis libros en lugar de los ocho de la tradición de los comentarios aristotélicos, se halla también en otro de los cursos anónimos conservado en el Archivo Nacional Histórico (Vol. 23, Fondo Varios, 1727) ${ }^{7}$, aunque los contenidos están dispuestos en otro orden.

En términos generales, el libro primero De composito naturali (ff. 1 - 92v), se estructura en cinco disputaciones. El Jesuita organiza con especial cuidado el estudio de cada uno de los elementos que lo conforman. Así, se centra, primero, en los principios intrínsecos del cuerpo natural en general y, luego, estudia con detención los elementos teóricos referidos al compuesto natural. De esta manera, trata sobre la materia prima, a la que denomina el primer principio del cuerpo natural y estudia qué es el apetito de la materia hacia las formas; si la materia prima apetece más a las formas más perfectas que a las menos perfectas; si la materia prima apetece a las formas corruptas bajo circunstancia de corrupción; si una forma una vez producida y destruída puede de modo natural reproducirse; $y$, finalmente, propone y examina algunas dudas para asegurar una comprensión completa del concepto de materia prima.

Después, aborda el estudio de la forma substancial o segundo principio del cuerpo natural. En este punto en especial se extiende y, entre otros temas, explica qué y cuántas son las formas substanciales; cuáles formas son creadas y cuáles educidas; si la forma es toda quididad o una esencia compuesta; si la materia prima puede tener de modo natural a la vez una doble forma no subordinada; y si en los seres vivientes se da una forma substancial de corporeidad subordinada a la forma viviente. Con posterioridad, Romo explica la unión entre la materia y la forma y, por último, las características del todo o compuesto. Hallamos que en cada una de las disputaciones indicadas, el jesuita incluye secciones destinadas a resolver con detalle las objeciones de los autores tomistas.

El libro segundo, De causis extrincesis corporis naturalis (ff. 93r-171v), se divide en cuatro disputaciones. Aquí, se dedica a estudiar detenidamente el papel de las distintas causas, insistiendo, en numerosas ocasiones, en las diferencias de interpretación con la escuela tomista. Se refiere a las causas en general (eficiente, material, formal, ejemplar y final), a la conexión de la causa con la posibilidad del efecto, a la causa eficiente increada (Dios y su concurrencia en diferentes efectos) y, por último, a la causa eficiente creada.

\footnotetext{
${ }^{7}$ Cf. Aravena Zamora (2015, p. 296).
} 
El libro tercero, De motu, actione, continuo et infinito (ff. 172r - 191v), se organiza en tres disputaciones: en la primera, se ocupa del movimiento y la acción, y en las otras dos, de la composición de cada uno de los elementos anunciados. De igual modo, en el libro cuarto, De loco et tempore (ff. 192r - 218v), se ocupa en dos disputaciones del lugar y el tiempo, mientras que en el quinto, De generatione et corruptione (ff. 219r - 223v), se refiere a la generación substancial y accidental, a la corrupción opuesta a la generación y a otros aspectos sobre las cualidades accidentales.

Por último, en el sexto y último libro, De mundo, caelo et metheoris (ff. $224 \mathrm{r}-227 \mathrm{v}$ ), aborda, en una única disputación, el estudio detenido del mundo, los globos celestes y el equilibrio requerido en la mezcla de los elementos para la conformación de dichos cuerpos.

\section{Física contra Tomistas}

Primero, quisiéramos aclarar la afirmación sobre la filiación tomista que los jesuitas Hanisch y Astorquiza atribuyen a la obra de Romo. Pues, en efecto, ambos desatienden una abreviatura clave en el lomo del volumen $(\supset \alpha=$ contra $)$ y la identifican como Física tomista. No obstante, como pretendemos demostrar, la obra constituye la reacción del maestro jesuita contra los seguidores del Aquinate en determinados temas de importancia doctrinal.

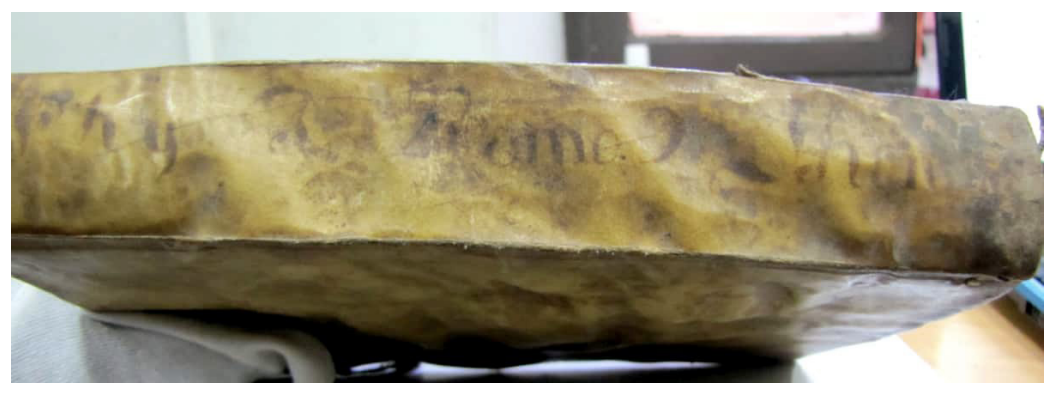

Detalle del lomo del volumen con el título "Phys. P. Romo $\supset \alpha$ Thom".

Romo declara al comienzo del proemio que "feliz es quien puede conocer las causas de las cosas", verso de Virgilio que atribuye al carmelita italiano Baptista Mantuanus (1447-1516), y, por ello, su obra considerará el estudio, examen e investigación en torno a las causas, efectos y milagros de la naturaleza. Añade además que el término "Física" deriva de la palabra griega "Physis" y, por esa razón, la disciplina recibe también el nombre de "filosofía natural" y de "fisiologia".

\footnotetext{
${ }^{8}$ Cf. Romo, Physica contra Thomistas, 1739, Prooemium, f. 1r.
} 
De acuerdo con Romo, el objeto material de la física es "el cuerpo natural completo con todas sus propiedades, pasiones, efectos y causas intrínsecas y extrínsecas"; su objeto formal es "cualquier concreto, o compuesto físico, ya substancial, ya accidental"; y su objeto de atribución es "el ente natural o compuesto substancial completo”, al que se llama también ente móvil, cuerpo natural, substancia natural o cuerpo substancial completo. Además, indica que "la Física es una ciencia por sí misma”, pues evidentemente demuestra patrones y propiedades; que "es de modo adecuado intrínsecamente especulativa"; y, por último, que "la Física no consiste en simples cualidades, sino que está compuesta por muchos hábitos distintos de modo real entre sí" 9 .

Como hemos adelantado, en la primera disputación del libro primero, Romo estudia los principios intrínsecos del cuerpo natural en general. Para ello, explica primero qué son los principios, distinguiendo los distintos modos en que pueden entenderse. Primero, en un sentido amplio, cómo aquello que origina alguna cosa, en el modo en que se dice que la aurora es el principio del día. Luego, cómo las razones primeras evidentes per se, así, por ejemplo, lo es el principio de no-contradicción, según el que es imposible que algo sea y no sea a la vez; y, también, -dice- cómo los cuatro elementos, que son llamados los principios de la naturaleza ${ }^{10}$.

El Jesuita propone en este punto la definición de Aristóteles (Met., V, 1, 13-16): "Principium unde aliquid fit, est, aut cognoscitur", en la que -precisahay que entender por "principio que hace [algo de una cosa]", los principios efectivos y extrínsecos; por "principio que es", los principios constitutivos e intrínsecos de un cuerpo natural; y por "principio por lo que algo resulta cognoscible”, los principios directivos e iluminativos. Agrega que la definición de "principio" dada por Santo Tomás es mejor y más universal: "aquello de lo que algo procede de algún modo". A partir de ella, explica que los filósofos más destacados, junto con Suárez, afirman que "el principio es aquello a partir de lo que algo es, o aquello que comunica el ser en algo", de donde se sigue la definición según la cual "el principio del ente natural es aquello a partir de lo que es el ente natural, o aquello que comunica el ser al ente natural"11.

De acuerdo con esto, afirma Romo, consta que se requieren al menos dos fundamentos para establecer los principios. Primero, que aquello que sea principio de algo tenga prioridad en el tiempo, como un padre respecto

\footnotetext{
${ }^{9}$ Cf. Romo, Physica contra Thomistas, 1739, Prooemium, f. 1v.

${ }^{10}$ Cf. Romo, Physica contra Thomistas, 1739, L. I, disp. $1^{\text {a }}$, sect. 1 , ff. 1v-2r.

${ }^{11}$ Romo, Physica contra Thomistas, 1739, L. I, disp. 1 a , sect. 1, f. 2r. Cf. Santo Tomás, Summa Theologiae, $\mathrm{I}^{\mathrm{a}}, \mathrm{q}^{\mathrm{o}} .33$, art. $1^{\mathrm{o}}$.
} 
de su hijo, o prioridad en su composición. Y, segundo, que se dé alguna dependencia entre el principio y el término o, en otras palabras, entre lo principiado y su fundamento ${ }^{12}$.

Luego, explica que un ente, un cuerpo o un compuesto natural no es otra cosa que el compuesto substancial consistente en la unión entre la materia y las formas substanciales. Así, distingue entre el compuesto in facto esse y el compuesto in fieri. Define al primero de este modo: "es el mismo compuesto entitativa y substancialmente acabado y además permanente y substancialmente constituido", mientras que el segundo "es la acción o producción del mismo compuesto natural"13. Lo anterior afirma la opinión común en conformidad con Suárez, Arriaga, Oviedo y muchos otros autores jesuitas. Más adelante, se refiere con detalle a los otros modos en que puede considerarse un cuerpo natural, a saber: in ratione incipientis esse, in esse mutati, in esse conversi, in esse precise generati e in ratione desinentis esse ${ }^{14}$.

En la segunda disputación del libro primero, acerca de la materia prima, Romo estudia detenidamente sobre este primer principio del cuerpo natural. Aquí, explica qué es la materia prima, cuya existencia -dice- ha sido juzgada como cierta por todos los filósofos: los peripatéticos con Aristóteles, Santo Tomás, el Doctor Sutil y, por cierto, Suárez. Al respecto escribe:

...nam materiam primam existere non obscure convincunt continuae generationes et corruptiones, frequentesque mutationes et conversiones substantiales, quas passim fieri experimur; quotidie enim videmus ex ligno fieri ignem, et ignem transire in cineres: ergo quando ignis destruit lignum, remanet aliquod ligni, sed non remanet forma ligni: ergo remanet aliquod subiectum, quod nunc recipit formam ignis et antea recipiebat formam ligni, sed hoc quod remanet et recipit formas non est aliud nisi materia prima: ergo haec est, seu existit in rerum natura ${ }^{15}$.

Se desprende de esto que, a partir de la evidencia del cambio substancial, se deduce también la existencia y la naturaleza de la materia prima. Pues, todo cambio supone un sujeto común a ambos términos, vale decir, supone un substrato substancial: la materia prima. En efecto, ella es el constitutivo esencial de los cuerpos, que permanece a través de los incesantes cambios substanciales, y que es además indeterminada, pues sin ser un cuerpo puede

\footnotetext{
${ }^{12}$ Cf. Romo, Physica contra Thomistas, 1739, L. I, disp. 1a , sect. 1, f. 2r.

${ }^{13}$ Romo, Physica contra Thomistas, 1739, L. I, disp. 1a , sect. 2, f. $2 \mathrm{v}$.

${ }^{14}$ Cf. Romo, Physica contra Thomistas, 1739, L. I, disp. $1^{\text {a }}$, sect. 4-9, ff. 5r-11r.

${ }^{15}$ Romo, Physica contra Thomistas, 1739, L. I, disp. 2a, sect. 1, f. 13r.
} 
llegar a ser cualquiera de ellos. Por esta razón, requiere de la forma como otro constitutivo determinante, ya que es la forma substancial la que en un cuerpo natural determina a la materia a ser rectangular o circular, animada o inanimada, etc. Sin embargo, no se debe concebir a la materia prima como una parte de los cuerpos, sino, más bien, como un principio de determinabilidad de ellos ${ }^{16}$.

En este contexto, el Jesuita desarrolla en detalle diferentes matices teóricos de este fundamental concepto metafísico. De este modo, se propone aclarar algunas dudas en torno a la materia prima, como, por ejemplo, si ella es sujeto antes que la forma en el compuesto. Al respecto, y siguiendo expresamente - dice- al Aquinate, al Eximio y al común de los filósofos, argumenta que la materia prima es sujeto antes que la forma, puesto que está ordenada a la recepción, vale decir, es una potencia receptiva.

Romo aborda también con detención otras importantes cuestiones doctrinales relacionadas con la concepción metafísica de la materia prima. Entre ellas, cabe destacar las siguientes: si la potencia receptiva de las formas se identifica realmente con la materia prima; si y cómo la materia prima es pura potencia; si la materia prima existe por existencia propia o por la existencia de una forma; si y cómo la materia prima depende de una forma; $\mathrm{y}$, también, si la materia prima puede existir por disposición divina sin alguna forma substancial ${ }^{17}$.

Grosso modo, en estos temas la interpretación suareciana sostiene, por un lado, que la materia prima tiene su esencia actual distinta de la de la forma, pero en cierto modo dependiente de ella. Pues, la materia prima que existe en el compuesto tiene alguna esencia real, que es distinta de la forma, y mediante ella tiene su perfección esencial propia y distinta de la que la forma le confiere. Dicha esencia actual de la materia depende trascendentalmente de la forma por el mismo hecho de ser potencia, ya que toda potencia depende trascendentalmente de su acto. Y, por otro lado, de aquí se deriva también que la materia prima tiene su existencia propia distinta de la existencia de la forma, aunque dependiente de ella. Pues, la entidad de la existencia no añade nada real a la entidad de la esencia actual, por lo que la materia prima tiene su existencia propia distinta de la existencia de la forma ${ }^{18}$.

Es, justamente, en este último punto en donde se hallan expresamente las mayores diferencias teóricas del maestro jesuita con los autores tomistas, por lo que, dejando al margen otras interesantes cuestiones del curso, en lo que viene centraremos nuestro esfuerzo en este tema en especial.

\footnotetext{
${ }^{16}$ Cf. Zaffaroni (1949, p. 296).

${ }^{17}$ Romo, Physica contra Thomistas, 1739, L. I, disp. $2^{\mathrm{a}}$, sect. 1-10, ff. 15r-55r.

${ }^{18}$ Cf. Zaffaroni (1949, pp. 297-298).
} 
En efecto, uno de los aspectos más novedosos es que en sus páginas se plasma una de las controversias filosóficas más importantes en la historia de la disciplina, que generó interminables discusiones en Europa ${ }^{19} \mathrm{y}$, por lo que vemos expresado en el curso -ya desde el propio título- también en el quehacer filosófico de la época colonial chilena.

Desde esta perspectiva, la tesis suareciana de que no hay una distinción real entre esencia y existencia en lo seres finitos, concebidas estas como dos entidades distintas, generó interminables disputaciones en el ámbito filosófico europeo. Y, por cierto, esta problemática fundamental la hallamos expresada también en la Física contra Tomistas del maestro jesuita.

Sobre este punto, conviene recordar brevemente que a lo largo de la Disputación XXXI de las Disputationes Metaphysicae, el Eximio se ocupó fundamentalmente de las tres soluciones propuestas en la polémica de la esencia y la existencia. De esta manera, analizó si existía entre ellas una distinción real, una distinción modal o una distinción de simple razón. En este contexto, explicaba que la tesis de la distinción real de esencia y existencia es la que pasaba por ser la opinión de Santo Tomás y sus seguidores, por lo que intentó demostrar que "la esencia creada constituida actualmente fuera de las causas, no se distingue realmente de la existencia, de tal manera que sean dos cosas o entidades distintas" ${ }^{\prime 20}$.

De acuerdo con la interpretación de Suárez sobre dicha distinción tomista, no puede afirmarse que la esencia creada, una vez puesta en acto, es aún distinta de su existencia, como si la esencia y la existencia fueran dos entidades distintas. Pues, el ser de la esencia actual no necesita de la existencia, a la manera de un complemento, para ser actualmente. En otras palabras, la esencia es o bien actual o bien, posible; y la única diferencia entre ambas condiciones es que aquello que es actual es, en cambio, aquello que es solo posible no es. Por ello, señalar que una esencia es un verdadero ser actual, es afirmar que tal esencia es actualmente, vale decir, que existe.

Esta identificación suareciana entre esencia y existencia trae como consecuencia el concebir a todos los seres actuales simplemente como distintas esencias plenamente actualizadas. En este sentido, la existencia actual no podría agregar nada a un ser que ya existe, pues una cosa ya existente tendría que incluir otra cosa además de lo que es, lo que no tiene sentido ${ }^{21}$. Por esta razón, el ser actual no es más que un caso particular del ser posible y, en definitiva, ser

\footnotetext{
${ }^{19}$ Cf. Gilson (1972, p. 150 ss).

${ }^{20}$ Suárez, Disputationes Metaphysicae, D. XXXI, s. VI, art. 1.

${ }^{21}$ Cf. Suárez, Disputationes Metaphysicae, D. XXXI, s. V, art. 3.
} 
en acto es lo mismo que ser existente ${ }^{22}$. En términos más precisos, el concebir la identidad entre esencia y existencia en los seres creados implica, por cierto, que estas no son dos cosas distintas, sino un todo único, per se unum.

A partir de lo anterior, deviene una concepción metafísica de las esencias, para la que las existencias no constituyen un objeto de estudio relevante, pues, para todos los filósofos cristianos, una creatura no existe por derecho propio, sino que necesita recibir la existencia de $\operatorname{Dios}^{23}$. Así, la esencia es primera en orden de dignidad y de prioridad, porque ella constituye, por un lado, lo que es una cosa en tanto que una cosa y, por otro, en tanto que tal cosa; puesto que ens y res son, para el Eximio, términos equivalentes ${ }^{24}$.

Esto es importante aquí, pues, consecuentemente desde esta perspectiva, la materia prima es el substrato necesario para el cambio substancial, como un constitutivo intrínseco del ser corpóreo. Además, se le concibe como única y común a todos los cuerpos naturales, excluyendo en sí misma toda composición física de acto y potencia, pero admitiendo una composición metafísica, según la cual tiene un acto entitativo que le otorga su esencia real propia y su existencia también propia. Y, por último, la materia prima creada por Dios es una entidad, vale decir, una esencia completamente real, que en cierto sentido tiene por sí misma un acto de existencia de manera independiente de la forma ${ }^{25}$.

Por el contrario, distinta es la interpretación entre los autores tomistas. Como lo apunta Kuri Camacho "en Santo Tomás el acto creador no tiene por término ni la materia prima ni la forma sustancial por separado, sino la substancia corpórea, el compuesto, el cual no es ni la forma sola ni la materia sola, sino un resultado de la unión de ambas a la vez"26. Y dicha unión, para el Angélico, se deriva de la distinción real entre la esencia y la existencia. Desde este punto de vista entonces, Dios no crea la materia prima por un lado y la forma, por otro; ni tampoco la materia prima antes que la forma ${ }^{27}$.

En suma, el meollo de este trascendental problema es explicar si la actualidad total de un ser real puede ser definida completamente en el solo plano de la esencia o, de otra manera, si la existencia es el acto último de la esencia en la línea de la misma esencia ${ }^{28}$.

${ }^{22}$ Cf. Suárez, Disputationes Metaphysicae, D. XXXI, s. IV, art. 6.

${ }^{23}$ Kuri Camacho (1995, p. 78).

${ }^{24}$ Cf. Suárez, Disputationes Metaphysicae, D. II, s. IV, art. 14; D. III, ss. III y IV. Con respecto a las críticas de la interpretación "esencialista" de Gilson sobre este aspecto de la filosofía de Suárez, véase Salas (2015, pp. 336-362).

${ }^{25}$ Cf. Zaffaroni (1949, p. 299).

${ }^{26}$ Kuri Camacho (1995, p. 75).

${ }^{27}$ Cf. Gilson (1989, pp. 343-363).

${ }^{28}$ Cf. Gilson (1972, p. 150). 
Volviendo al texto de Romo, en torno a esta controversia, el Jesuita expone primero la opinión que sostiene que la materia prima depende esencialmente de la forma, de modo que ni por medio de una disposición divina puede existir sin alguna forma, lo que es afirmado -con muy pocas excepciones, dice- por la totalidad de la escuela tomista. En segundo lugar, presenta la opinión que afirma que, por disposición divina, la materia prima puede existir sin alguna forma. Este posición no implica, pues, una dependencia esencial de la materia con la forma. Esta visión la comparten San Agustín, Ambrosio, Basilio y Crisóstomo, citados por el Aquinate, y además los escotistas con el Sutil y los jesuitas con Suárez, a quien -explica- siguen muchos autores externos a la Compañía, y también algunos tomistas como Medina, Báñez, Soto y Vitoria ${ }^{29}$.

Para probar la primera conclusión, Romo señala cinco argumentos, que nos limitaremos aquí solamente a enunciar. Primero, que un cuerpo, por disposición divina, puede existir sin extensión local y ubicación circunscriptiva aunque su naturaleza exija el existir extensa y localmente. Por la misma razón, la materia podría existir sin una forma por disposición divina, aunque su naturaleza exija el existir con alguna forma. En segundo lugar, que de igual manera depende la forma material de la materia como la materia depende de una forma, ya material, ya espiritual. Pero, como afirman los tomistas, la forma material puede existir sin la materia por disposición divina, por lo que, por esta misma razón, también podría la materia existir sin una forma. El tercer argumento recurre a un pasaje de la Epístola a los Corintios (15:53), y el cuarto a Apocalipsis 6: 12 (sol factus est niger), según el cual el sol puede existir sin luz por una disposición divina y, por una razón similar, la materia puede existir sin una forma. Y, por último, a semejanza de lo que ocurre en la Eucaristía, en la que por disposición divina los accidentes existen sin un sujeto, la materia puede por esta misma razón existir sin una forma ${ }^{30}$.

Por otra parte, como prueba de la segunda conclusión, Romo invierte los argumentos de los tomistas, lo que le permite, primero, afirmar que, aunque la materia exista naturalmente por la existencia de la forma, puede existir por disposición divina por la existencia de otro ente que no sea una forma. Así, de acuerdo con los seguidores del Angélico, la humanidad de Cristo, vale decir, la

\footnotetext{
${ }^{29}$ Romo, Physica contra Thomistas, 1739, L. I, disp. 2a, sect. 10, f. 32r: "Prima sententia defendit materiam primam dependere essentialiter a forma ita ut nec divinitus possit esse sine omni forma: ita universa schola thomistica paucis exceptis. Secunda sententia defendit materiam primam posse divinitus existere sine omni forma, ideoque nec dicere essentialem dependentiam a forma: ita Divus Agustinus, Ambrosius, Basilius et Chrisostomus citati a Divo Thoma; item etiam Schotisticam cum suo subtili, et nostri cum Eximio, quos sequuntur plures ex externis, et ex Thomistii Medina, Bañez, Soto et Victoria...”.

${ }^{30}$ Cf. Romo, Physica contra Thomistas, 1739, L. I, disp. 2a, sect. 10, f. 32r.
} 
materia prima de su humanidad, existe por medio de la existencia del Verbo. Luego, explica que es tan propio de la naturaleza humana el subsistir por una subsistencia creada como es propio de la materia prima el existir por medio de la existencia de la forma. Sin embargo, advierte, la naturaleza humana puede subsistir, por una disposición divina, por medio de una subsistencia que no sea creada y, por la misma razón, la materia puede existir por una subsistencia que no sea una forma. Así, entonces, por disposición divina, la materia puede existir sin una forma.

El Jesuita indica que la existencia de la materia y la forma, según los tomistas, es primariamente la existencia del todo y, en segundo lugar, es la existencia de las partes, es decir, de la materia y de la forma tomadas por separado. No obstante, expone que una parte, por ejemplo, la forma, puede existir por disposición divina antes que el todo, como lo demuestra y consta evidentemente en el alma racional. Por tanto, también puede existir la materia prima sin la forma por disposición divina.

Romo explica lo anterior agregando que, aunque la forma exista por medio de la existencia de la materia, para los tomistas la forma puede existir sin materia por una disposición divina: por tanto puede también esta por una disposición divina existir sin la forma. De aquí que, por paridad de argumento, aunque la materia exista por medio de la existencia de la forma, podría por una disposición divina existir sin alguna forma. Añade que el antecedente es cierto, puesto que una es la existencia de la materia y de la forma en opinión de los tomistas, y que la consecuencia también parece cierta por paridad de argumento ${ }^{31}$.

Posteriormente, en una sección exclusiva, se dedica con especial cuidado a resolver distintas objeciones de los tomistas relevantes en este punto de la controversia. En torno a ello, aunque sea brevemente, rescataremos algunos pasajes de la enseñanza filosófica del maestro jesuita.

Así, hallamos que, para polemizar contra los seguidores del Aquinate, desarrolla la interpretación suareciana sobre las causas intrínsecas, esto es, sobre aquellas causas que constituyen sus efectos permaneciendo en ellos. Vale decir, estas causas intrínsecas se corresponden con las nociones de materia y forma.

\footnotetext{
${ }^{31}$ Romo, Physica contra Thomistas, 1739, L. I, disp. 2a, sect. 10, f. 32v: “...licet forma existat per existentiam materiae, potest per vos forma divinitus existere sine materia: ergo a pari licet materia existat per existentiam formae, poterit divinitus materia existere sine forma. Antecedens est certum, quia una est existentia materiae et formae in sententia Thomistarum. Consequentia etiam videtur certa a paritate rationis".
} 
En este contexto, acerca de la causa formal en particular enseña que esta se dice tal con respecto a un efecto formal por ella constituido. Por ello la forma no es la causa formal de la materia, puesto que esta no está constituida por la forma, ya que es el compuesto el efecto formal constituido por la forma; y lo demuestra ejemplificando que el blanco no es la causa formal de una pared, sino de su color blanco en concreto, que está constituido por la blancura. Agrega que, sin embargo, el informar no es suficiente para que haya causa formal, pues, de otra manera un accidente sería causa formal de la substancia que informa por medio de la unión; mas para informar, se requiere que sea una parte constitutiva y determinativa con prioridad al mismo efecto formal, del que es causa formal, que en unión tiene la forma respecto del compuesto, pero no respecto de la materia. En este contexto, desliza una crítica a los autores tomistas cuando escribe que "...praeterea licet ad causam formalem sufficeret informare adhuc nihil convincerent Thomistae, quia in hoc casu falsum est quod res non possit existere sine sua causa formali, nam partes informantur per albidinem et tamen potest existere sine alia quod etiam in aliis patet" ${ }^{\prime 32}$.

Luego, puntualiza, por un lado, que la forma no es la causa formal de la materia, puesto que no es una parte constitutiva y determinativa ni tampoco tiene prioridad ante la materia misma; $y$, por otro, que la materia bien puede ser la causa material de la forma, puesto que es anterior tanto como de ella depende lo que tiene forma. En cambio, la forma no puede ser anterior a la materia y, por ello, de ningún modo puede ser la causa formal de la materia ${ }^{33}$.

Más adelante, agrega que la materia prima no depende esencialmente de la forma, sino solo de modo natural (naturaliter), puesto que no depende de ella para existir por sí, sino en tanto que existe en su ser natural o con su complemento natural, el que por disposición divina puede ser privado, así como entre los tomistas - dice- la forma substancial material puede por disposición divina privarse del complemento substancial de la materia y sin él, privada de ese modo existiría por disposición divina ${ }^{34}$.

A partir de ello, Romo enseña, como conclusión del primer libro, que la materia prima esencialmente no es una parte actual, sino solo aptitudinal, y su esencia no es ser en acto con el compuesto con el que se une, sino solo puede

\footnotetext{
${ }^{32}$ Romo, Physica contra Thomistas, 1739, L. I, disp. 2a, sect. 11, f. 34v.

${ }^{33}$ Cf. Romo, Physica contra Thomistas, 1739, L. I, disp. 2a, sect. 11, f. 34v.

${ }^{34}$ Romo, Physica contra Thomistas, 1739, L. I, disp. 2a, sect. 11, f. 35v: "At vero materia prima non dependet essentialiter a forma, sed solum naturaliter, cum non dependeat ab illa ut ab se existat, sed ut existat in suo esse naturali seu cum suo naturali complemento, quo potest divinitus privari, sicut apud ipsos adversarios forma substantialis materialis potest divinitus privari complemento substantiali materiae, et sine illo sic privata divinitus existeret: ergo a pari in nostro casu”.
} 
ser con ella, como es evidente en el alma racional y en otras formas. Y, por otra parte, señala que, de acuerdo con los principios suarecianos, Dios no puede suplir el defecto de la causa formal en el mismo género formal, puesto que la causa formal es parte esencial del efecto formal, y, por ello, Dios no puede hacer que se dé el blanco sin la blancura, ni el entendimiento sin la intelección. En cambio, para los autores tomistas, quienes dicen que basta informar a la causa formal, Dios puede suplir el defecto de la causa formal, cuando esta no es única, sino múltiple. En este caso, la existencia de la forma no sería la única causa formal de la materia, puesto que esta podría existir por disposición divina por medio de otra existencia, ya substancial, ya accidental, ya natural, ya sobrenatural, ya creada, o ya increada ${ }^{35}$.

Por otro lado, otra trascendental discrepancia con los seguidores del Aquinate la hallamos en torno a la intepretación de la doctrina aristótelica sobre la reciprocidad de las causas ${ }^{36}$. En este contexto, Romo expone como una réplica tomista la interpretación a un pasaje del segundo libro de la Física, según el cual “causae sunt sibi invicem causae” y, por tanto, la materia y la forma se causan recíprocamente. Se deriva de aquí, además, que así como la materia causa materialmente a la forma, del mismo modo la forma causa formalmente a la materia. No obstante, aclara que, para los jesuitas, la doctrina del Estagirita es evidentemente clarísima, mas no así para los seguidores del Angélico, quienes - dice- le son menos fieles y truncan su autoridad. Así, las palabras del Filósofo respecto de las causas "sunt autem quaedam ad invicem causae, sed non eodem modo, sed hoc quidem ut finis, illud autem ut prium motus", es manifiesto que no se refieren a toda causa, sino a la causa final y efectiva. Explica lo anterior debido a que la causa final puede ser causa de sí, ya que la causa final no requiere precedencia en ejecución, sino solo en intención, mientras que las causas físicas requieren precedencia en ejecución ${ }^{37}$.

\footnotetext{
${ }^{35}$ Romo, Physica contra Thomistas, 1739, L. I, disp. 2a, sect. 11, f. 36v: “...quod materia prima essentialiter non est pars actualis, sed solum aptitudinalis, de cuius essentia non est actu esse cum composite cum qua componit, sed solum posse esse cum illa ut patet in anima rationali, et aliis formis. Inferes $2^{\circ}$, quod in nostris principiis Deus non potest suplere defectum causae formalis in eodem genere formali, cum iuxta nos causa formalis sit pars essentialis effectus formalis, et ideo dicimus quod non potest Deus facere ut detur album sine albedine, nec intelligens sine intellectione, ut vero in principiis Thomistarum, qui dicunt suficere informare ad causam formalem, potest Deus suplere defectum causae formalis, quando haec non est unica, sed multiplex, ut accideret in nostro casu in quo existentia formae non esset unica causa formalis materiae, cum haec posset divinitus existere per aliam existentiam sive substantialem, sive accidentalem, sive naturalem, sive supernaturalem, sive creatam, sive increatam, ut supra diximus".

${ }^{36}$ Cf. Falgueras (1997, p. 39 ss).

${ }^{37}$ Cf. Romo, Physica contra Thomistas, 1739, L. I, disp. 2a, sect. 11, f. 34v.
} 
Por último, otro interesante aspecto que quisiéramos rescatar brevemente, pues constituye otra arista de la controversia filosófica que nos interesa mostrar, es la refutación expresa de algunas ideas tanto de autores tomistas como también de otros autores de la misma Compañía. En este contexto, Romo polemiza frecuentemente con maestros como Juan Polanco S. J (1517-1576), Nicolás de Olea, S. J (1635-1705) y Jacinto de la Parra, O. $\mathrm{P}$ (1619-1684), entre otros. Por razones de espacio, nos limitaremos aquí a rescatar solo una de estas referencias.

Así, en un pasaje de la Física contra Tomistas, se refiere expresamente a los maestros Polanco y Parra, quienes - escribe- dicen que argumentan demostrativamente contra los autores de la Compañía en la discusión sobre si la materia puede existir por medio de una disposición divina. En este contexto, el Jesuita señala que "Si melius suam argumentationem considerassent Parra et Polancus, non illam dicerent demonstrationem, sed aeque vocationem, vel saltem demonstrationem risu dignam appellarent, ut patet manifeste ex retorsione sic formata in principiis Thomistarum..." 38 .

En esta polémica, Romo agrega que cualquier cosa se hace capaz de acto segundo lógico y entitativo por medio de un acto primero también lógico y entitativo, pero niega que se haga capaz de acto segundo lógico y entitativo por medio de un acto primero físico e informativo. Pues, la existencia es el acto segundo lógico y entitativo de la materia en existencia per se informable y la forma es el acto primero tambien lógico y entitativo de la materia en existencia per se informable. Sin embargo, la forma es también el acto primero físico informativo ${ }^{39}$.

Sobre este mismo punto explica que es cierto que una cosa, que esté en la misma línea con un acto primero, alcanza la capacidad de acto segundo a través de este acto primero. Así, la materia prima podría alcanzar la capacidad de acto segundo de la existencia, que es acto segundo lógico y entitativo, por un acto primero lógico y entitativo o por la potencia para existir realmente identificada con ella. No obstante, -dice- convencerá razonablemente que la materia no puede alcanzar la capacidad para existir por medio de la misma forma, puesto que esta no es acto primero lógico y entitativo, sino solo físico e informativo. Por lo tanto, puesto que el acto segundo de la existencia está en diversa línea que el acto primero de información, podría darse en la materia al menos por disposición divina el acto segundo de la existencia sin el acto primero de la información, y por ello, la materia podría por medio de una disposición divina

\footnotetext{
${ }^{38}$ Romo, Physica contra Thomistas, 1739, L. I, disp. 2a, sect. 11, f. 33v.

${ }^{39}$ Cf. Romo, Physica contra Thomistas, 1739, L. I, disp. $2^{\mathrm{a}}$, sect. 11, ff. 33v-34r.
} 
existir sin una forma. El Jesuita aclara nuevamente que aunque la materia no exista por medio de la existencia de la forma, por disposición divina puede existir por medio de otra existencia, ya natural, ya sobrenatural, ya creada, ya increada, como los tomistas dicen acerca de la existencia del Verbo ${ }^{40}$.

Con la finalidad de confirmar lo dicho, Romo insiste en que la existencia de la materia o acto segundo de la existencia no está en la misma línea con la forma o acto primero de información, puesto que la existencia de la materia está en línea entitativa informable mientras que la forma, solo en línea física informativa substancial. Por ello bien puede por disposición divina darse el acto segundo de la existencia de la materia sin el acto primero de la forma. Por último, precisa que la existencia de la materia no es un modo esencialmente adjunto a la forma, como es evidente en la existencia del Verbo que alcanza la existencia de la forma, y toda la humanidad de Cristo para los tomistas ${ }^{41}$.

\section{Conclusiones}

La filosofía de la época colonial chilena es un área aún muy poco explorada dentro de la disciplina. En efecto, gran parte de las obras manuscritas de este período se encuentran aún inéditas. Las razones de esto incluyen el deteriorado estado de conservación de muchos de los volúmenes, la dificultad para comprender el latín en el que fueron redactados los cursos, las profusas abreviaturas que dificultan la comprensión y la lectura de los contenidos, la complejidad de la caligrafía del copista, el conocimiento de los autores, de las disputaciones filosóficas y de los diferentes temas estudiados en las obras, etc.

En este sentido, la Física contra Tomistas de Lorenzo Romo es una obra que hasta este trabajo no había sido objeto de estudio. Más aún, en los pocos catálogos en los que era mencionada, aparecía con una filiación del todo contraria a su espíritu. Por ello, valía la pena profundizar en el estudio de alguno de sus aspectos y, aunque sea esta vez a través de un acercamiento exploratorio, ofrecemos material inédito acerca de una controversia filosófica en el Chile colonial.

De este modo, a partir del estudio de una parte de los contenidos del curso, hemos evidenciado que, en afinidad con lo que ocurría en otras latitudes de América, para estas alturas de mediados del XVIII se escuchaba con más frecuencia la voz de Francisco Suárez que la de Santo Tomás en los colegios

\footnotetext{
${ }^{40}$ Cf. Romo, Physica contra Thomistas, 1739, L. I, disp. 2a, sect. 11, f. 34r.

${ }^{41}$ Cf. Romo, Physica contra Thomistas, 1739, L. I, disp. $2^{\text {a }}$, sect. 11, f. 34r.
} 
jesuitas chilenos. Así, en sus enseñanzas, Romo sigue fielmente a Suárez en temas fundamentales, como, por ejemplo, en la total dependencia de las criaturas de Dios.

Por otro lado, consta que la controversia filosófica respecto de la diferencia real entre esencia y existencia, tan cultivada en el ámbito europeo, se dio también en el Chile colonial. En este sentido, creemos que los textos que hemos presentado así lo demuestran. Lo mismo podemos afirmar, por ejemplo, respecto a las interpretaciones suarecianas y tomistas de la teoría de las causas aristotélicas.

De acuerdo con todo lo anterior, al presentar material hasta ahora inédito, aspiramos primeramente a contribuir en la apertura de nuevas líneas de investigación en torno al quehacer filosófico de la época, a recuperar las obras y profesores del período y, en última instancia, a enriquecer la tradición filosófica nacional.

\section{Referencias}

Aravena Zamora, Abel (2015). "Manuscritos filosóficos coloniales conservados en el Archivo Nacional Histórico de Santiago de Chile". Revista Española de Filosofía Medieval, 22, pp. 289-305.

Aravena Zamora, Abel (2016a). Filosofía en el Chile colonial: el curso de animástica de fray Juan de Fuica". Anales del Seminario de Historia de la Filosofia, 33 (2), pp. 475-488.

Aravena Zamora, Abel (2016b). "Un curso de filosofía del Chile colonial: los Comentarios Acerca del alma de fray Juan de Fuica (1689)". Veritas. Revista de filosofía y teología, 35, pp. 81-98.

Astorquiza, Fernando (1982). Bio-Bibliografia de la Filosofia en Chile desde el siglo XVI hasta 1980. Santiago de Chile: Barcelona, Empresa Industrial Gráfica.

Contreras Gutiérrez, Alejandra (2014). "La enseñanza jesuita en Chile colonial: sus colegios, universidades y una aproximación a sus métodos y contenidos". Revista Historia de la Educación Latinoamericana, 16 (22), pp. 35-50. 
Enrich, Francisco S. J. (1891). Historia de la Compañía de Jesús en Chile. 2 volúmenes. Barcelona: Imprenta de Francisco Rosal.

Enrich, Francisco S. J. (S/F). Breves elogios de la virtud de algunos sugetos, que fueron parte de la Provincia del Chile. Archivo Jesuita de Santiago de Chile: Ms. 2/J/294 carp. 17.

Falgueras, Ignacio (1997). Crisis y Renovación de la Metafísica. Málaga: Servicio de Publicaciones de la Universidad de Málaga.

Gilson, Étienne (1972). L'Etre et l'essence. Paris: Vrin.

Gilson, Étienne (1989). El Tomismo. Introducción a la filosofía de Santo Tomás de Aquino. Pamplona: Ediciones Universidad de Navarra, S. A.

Guarda, Gabriel O. S. B. (1978). Historia Urbana del Reino de Chile. Santiago de Chile: Editorial Andrés Bello.

Hanisch, Walter S. J. (1974). Historia de la Compañía de Jesús en Chile. Buenos Aires: Editorial Francisco de Aguirre.

Hanisch, Walter S. J. (1963). En torno a la filosofía en Chile (1594-1810). Santiago de Chile: Ediciones Historia, Universidad Católica de Chile.

Jobet, Julio Cesar (1970). Doctrina y praxis de los educadores representativos chilenos. Santiago de Chile: Editorial Andrés Bello.

Kuri Camacho, Ramón (1995). “Algunas proposiciones filosóficas y teológicas que no se enseñaban en los colegios de la Compañía de Jesús: siglos XVII y XVIII". Anuario Saber Novohispano, pp. 69-112.

Quiles, Ismael S. J. (1953). "Manuscritos filosóficos de la época colonial en Chile". Ciencia y Fe, 34, pp. 39-61.

Redmond, Walter (1972). Bibliography of the philosophy in the Iberian colonies of America. The Hague: Martinus Nijhoff.

Romo, Lorenzo S. J. (1739). Physica contra Thomistas. Archivo Histórico Mercedario de Santiago de Chile: Ms. 
Salas, Victor (2015). "Between Thomism and Scotism: Francisco Suárez on the Analogy of Being”. En V. Salas y R. Fastiggi (Eds.), Companion to Francisco Suárez (pp. 336-362). Leiden: Brill.

Skariça, Mirko (2010). "Alonso Briceño. Apuntes para una historia de la filosofía en Chile”. La Cañada, 1, pp. 6-21.

Tampe, Eduardo S. J. (2008). Catálogo de Jesuitas de Chile (1593-1767). Catálogo de Regulares de la Compañía en el antiguo Reino de Chile $y$ en el destierro. Santiago de Chile: Ediciones Universidad Alberto Hurtado; Instituto de Historia de la Pontificia Universidad Católica de Chile; Centro de Investigaciones Diego Barros Arana.

Zaffaroni, Juan Carlos S. J. (1949). “La materia prima en Suárez”. En Actas del IV Centenario del nacimiento de Francisco Suárez (1548-1948) (pp. 291-306). Madrid: Dirección General de Propaganda I. 